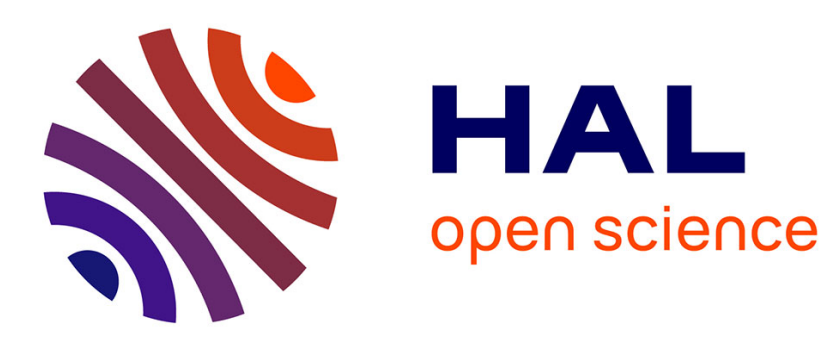

\title{
Déliaisons sociales et désubjectivation
}

Michèle Benhaïm

\section{To cite this version:}

Michèle Benhaïm. Déliaisons sociales et désubjectivation. Cliniques méditerranéennes, 2005, 72, pp.103 - 103. 10.3917/cm.072.0103 . hal-01429353

\section{HAL Id: hal-01429353 https://hal-amu.archives-ouvertes.fr/hal-01429353}

Submitted on 7 Jan 2017

HAL is a multi-disciplinary open access archive for the deposit and dissemination of scientific research documents, whether they are published or not. The documents may come from teaching and research institutions in France or abroad, or from public or private research centers.
L'archive ouverte pluridisciplinaire HAL, est destinée au dépôt et à la diffusion de documents scientifiques de niveau recherche, publiés ou non, émanant des établissements d'enseignement et de recherche français ou étrangers, des laboratoires publics ou privés. 


\title{
DÉLIAISONS SOCIALES ET DÉSUBJECTIVATION
}

Michèle Benhaïm

\author{
ERES | «Cliniques méditerranéennes »
}

2005/2 n $72 \mid$ pages 103 à 112

ISSN 0762-7491

ISBN 2749204054

Article disponible en ligne à l'adresse :

http://www.cairn.info/revue-cliniques-mediterraneennes-2005-2-page-103.htm

\section{Pour citer cet article :}

Michèle Benhaïm, «Déliaisons sociales et désubjectivation », Cliniques méditerranéennes 2005/2 ( $\mathrm{n}^{0} 72$ ), p. 103-112.

DOI $10.3917 / \mathrm{cm} .072 .0103$

Distribution électronique Cairn.info pour ERES.

(c) ERES. Tous droits réservés pour tous pays.

La reproduction ou représentation de cet article, notamment par photocopie, n'est autorisée que dans les limites des conditions générales d'utilisation du site ou, le cas échéant, des conditions générales de la licence souscrite par votre établissement. Toute autre reproduction ou représentation, en tout ou partie, sous quelque forme et de quelque manière que ce soit, est interdite sauf accord préalable et écrit de l'éditeur, en dehors des cas prévus par la législation en vigueur en France. Il est précisé que son stockage dans une base de données est également interdit. 


\section{Michèle Benhaim}

\section{Déliaisons sociales et désubjectivation}

\section{INTRODUCTION}

Si la souffrance psychique demeure le symptôme majeur de la précarité, nous sommes aujourd'hui convoqués à pratiquer dans des lieux (géographiques et psychiques) où le réel du lien social s'est substitué au réel du symptôme. En quoi la présence trop réelle de la maladie sociale, voire de la mort, nous oblige-t-elle à occuper ces terrains ?

Notre réflexion se donne pour objet de tenter de cerner ces lieux du réel, " ce qui subsiste hors de la symbolisation 1 » d'où pourrait surgir une " vérité » subjective.

Cette réflexion s'appuie sur une pratique psychothérapeutique d'analyste en CHRS (Centre d'hébergement et de réinsertion sociale) et à Médecins du monde. Des temps de consultation sont offerts dans le premier lieu à des jeunes de 18 à 25 ans désinsérés et ayant des parcours de vie jalonnés de ruptures (familiales, scolaires, sociales), de chaos (maltraitances, incestes, voire personnes étrangères victimes de tortures), et à des patients infectés ou coinfectés par le VIH et/ou le VHC ${ }^{2}$. À Médecins du monde, il s'agit d'un travail de rue ou de consultations dans lequel la situation inverse les enjeux relationnels ou transférentiels et où l'hypothèse qui soutient cette pratique « d'aller à la rencontre de » repose sur l'idée que faire une offre de parole a pour effet d'instituer l'autre comme sujet de demande.

Il s'agira donc de décrire les processus psychiques à l'œuvre dans le champ du réel social, puis d'en articuler certains aspects à la question du sujet. Comment faire que du sujet fonctionne là où du réel est à l'œuvre?

Michèle Benhaim, maître de conférences en psychopathologie clinique et psychanalyse, université de Provence, Centre des lettres et sciences humaines, psychanalyste ; 8 rue Alfred de Musset, 13006 Marseille. 1. J. Lacan, Écrits, Paris, Le Seuil, 1966.

2. Virus de l'immunodéficience humaine et virus de l'hépatite $C$. 
Si la fonction paternelle n'est plus assurée, et si le nom du père est ce qui permet à la mère d'exercer sa " fonction » alors il n'y a plus de "fonction » maternelle, fonction que l'on pourrait sommairement définir comme le passage d'une mère symbolique détentrice de l'objet réel à une mère réelle faisant fonction de "pourvoyeuse de signifiants ", ce moment où donner ce qu'elle a n'est plus l'enjeu et où elle donne ce qu'elle n'a pas, de l'amour. Ce serait peut-être plus juste que de réduire la question au seul déclin paternel qui laisserait le champ envahi par le registre d'un archaïque maternel.

Quoi qu'il en soit, c'est bien ce dont témoigne une clinique des exclusions articulée à ces problématiques adolescentes qui nous désemparent : $c^{\prime}$ est que la mère n'assure plus sa fonction lorsque jouissance et loi sont désarrimées, ce qui en résulte, pour ces adolescents, étant le refus de toute loi, lorsque la jouissance dans l'ordre sexuel n'est plus sublimée, lorsqu'elle n'est plus nouée au fantasme. "Ce qui lie les hommes entre eux, ce qui les oppose, est précisément à motiver de ce dont nous essayons pour l'instant d'articuler la logique 3 », nous dit Lacan, évoquant la logique du fantasme. Au sein de ce triple clivage, le réel social prend forme. Comment le traiter ?

Ainsi, nous nous appuierons sur une " clinique du réel » au sens où Ginette Raimbault évoquait ce que contenait d'impensable et par conséquent d'innommable, la mort d'un enfant où les effets de la maladie grave, au sens bien sûr où Lacan nous parle de ce registre hors-sens, qui ne se laisse pas résorber dans le signifiant, champ que ma réflexion m'amène à nommer clinique des «métamorphoses du réel » et qui relève :

- soit de situations où rien ne s'énonce du sujet (l'en deçà de la demande) et où je fais l'hypothèse clinique qu'il s'agit là, dans un premier temps, de faire justement émerger du réel, et, dans un second temps, de faire avec ce nonsymbolique, du "symbolique », sur le modèle du « praticable 4 » à l'œuvre dans les psychothérapies de patients schizophrènes : non-dupes du discours, les sujets demeurent dans l'errance d'une absence d'inscription. La rencontre transférentielle se soutiendra alors de ce quelque chose qui fait mal et qui viendra bousculer le déni.

- soit de cas de figure où le réel est un enjeu pour ce que le sujet va engager dans le transfert mais ne s'inscrit pas, par définition, comme signifiant possible (et ce, sous l'emprise de mécanismes de clivage). L'hypothèse clinique repose alors sur une construction du «traumatisme », la construction d'une fiction qui contribuerait, dans un premier temps, à border une jouissance informe, celle qui se passe de la nécessité du symptôme.

3. J. Lacan, 1996-1967, Séminaire La logique du fantasme, inédit.

4. Expression de Ginette Michaux, à peu près équivalente à la notion de « suppléance » chez Lacan. 
Nous verrons en conclusion comment, lorsque le sujet cesse de jouir du réel pour se mettre à jouir dans une plainte adressée, nous pouvons supposer qu'est en travail un mécanisme de substitution du réel du symptôme au réel social.

Reste à délivrer de la vérité de ce symptôme, entrevu alors comme une nécessité pouvant faire solution, un langage.

PROCESSUS PSYCHIQUES DU RÉEL SOCIAL

Quand Lacan énonce « l'inconscient c'est le politique » (il dit aussi « l'inconscient c'est le social »), il évoque la soumission du sujet au S1 parallèle au S1 qui gouverne le social, autrement dit il souligne la prise du sujet, au mieux, dans le désir de l'Autre social, voire aujourd'hui, au pire, dans sa jouissance.

Pour s'adapter à la réalité extérieure quelle qu'elle soit, le sujet doit pouvoir se confronter à sa réalité psychique, et pour ce, extraire de son Idéal du Moi un trait identificatoire particulier dont il fera son mode d'être au monde, à l'autre, sa manière singulière de s'inscrire dans le lien social, ou encore son mode spécifique de jouissance. Ce qui fait de la névrose, du symptôme en l'occurrence, un constituant à part entière du lien social.

Lien social ne prenant sens dans notre réflexion qu'articulé très étroitement à l'aspect symbolique de ce dit lien, c'est-à-dire au langage, à la pacification de la civilisation, à ce qui dans la relation intersubjective permet l'être-ensemble. Au sens où d'aucuns parlent aujourd'hui de " déchirures du tissu symbolique », et que je vais tenter de décliner comme autant de métamorphoses du réel conjuguées à ce que le lien social, faute d'être lui-même contenu dans un ordre symbolique, ne contient plus, en l'occurrence, la haine comme une des figures de la pulsion de mort. « La haine récuse l'appareil de langage... [elle]... fait advenir le réel en voulant l'abolir 5 », nous dit Roland Gori.

Ici, le constat clinique révèle que les sujets les plus « hors-lieu » sont les sujets les plus en proie à une errance subjective, les sujets réagissant, au mieux, au coup par coup au réel, au pire, exilés de leur propre langue, c'està-dire du symbolique ; ce que le social agit à cet endroit c'est d'une part accentuer les manifestations de cette condition psychique, $\mathrm{d}^{\prime}$ autre part fragiliser les liens sociaux qui, s'ils subsistent, sont extrêmement vulnérables et se délient. Au croisement ou au cœur de cet indénouable, le sujet déambule, nous opposant, au mieux, la répétition de ses passages à l'acte, au pire, son silence, un silence de sidération, un silence de mort...

5. R. Gori, «L'enfant mort des passions », Cliniques méditerranéennes, 70, 2004, 45-58. 
Comment favoriser la création de ce point de capiton qui permettrait au sujet d'y ancrer sa jouissance, et ce, non pas dans un semblant d'identité groupale, caractérisé par les particularités exclusives d'un mode de jouissance, mais dans une dimension singulière?

Ce qui semble venir percuter et redoubler de violence les souffrances psychiques actuelles c'est la question, déjà conflictuelle au niveau du sujet, du sexe et de ce «maître absolu 6 » qu'est la mort, sachant que ce qui en tient lieu est toujours menacé de déni.

Le sida a largement témoigné, dans ses déploiements imaginaires, des occurrences du réel dans le champ de la sexualité. Du côté de la mort, avant de développer notre propos, posons que la face mortelle de la pulsion, censée demeurer «muette », est le nœud de notre clinique.

La pulsion de mort, telle que Freud la découvre peut réduire l'individu à « néant ».

Ce que Lacan reprend en évoquant l'« assomption par l'homme de son déchirement originel par quoi on peut dire qu'à chaque instant il constitue son monde par son suicide 7 ».

La dissociation des pulsions de vie et des pulsions de mort est lourde de pathologies, leur désintrication conduit à la mort, voire à la transmettre.

Ce néant nous éloigne, pour le moins, de l'« être » en jeu dans le processus identificatoire évoqué plus haut.

Il faudra la patience d'une longue perlaboration, souvent douloureuse, toujours délicate, pour symboliser ce réel, c'est-à-dire pour symboliser la destruction même des conditions de la symbolisation.

Autrement dit, pour restaurer la dimension symbolique de ce que nous nommons « lien social », qui en garantit l'essence et qui prend aujourd'hui, au regard de la pulsion de mort, la figure d'un lien pervers, il nous faut élaborer non seulement les effets sur le sujet de la perversion du lien qu'il habite, mais la structure même de ce lien devenu mortifère. Énoncer ainsi les choses situe le lien social non plus dans une précarité dans laquelle il évoluait, certes, il y a encore quelques années, mais dans un champ véritablement psychopathologique, dans lequel un lien qui s'organise dans une sorte de culte de la souffrance, s'apparente à une pathologie de la relation à l'objet.

Et en effet, ce à quoi la clinique nous confronte, ce sont des sujets qui sont dans le manque réel, ce qui a pour effet d'inscrire les réponses qui leur sont proposées du côté d'objets réparateurs. Là, le plus souvent le sujet se dérobe et laisse les équipes sanitaires et sociales dans des positions dépressives relatives à leur sentiment d'impuissance. Ici tout le monde est captif

6. J. Lacan, Écrits, Paris, Le Seuil, 1966.

7. Ibid. 
d'un innommé et s'épuise ou se désertifie davantage. Il nous faut alors rétablir que le réel nous convoque non pas du côté d'une impuissance (imaginaire parce que corrélative de son pendant, la toute-puissance) mais du côté d'un impossible, celui qui régit le fonctionnement du sujet, la version structurelle du manque, nous souvenant que seulement de ce dernier peut naître le désir. L'impuissance dénie le réel, l'impossible, au contraire, le recouvre.

$C^{\prime}$ est ce qu'entendait peut-être Lacan lorsqu'il enjoignait la psychanalyse à « contrer » le réel, c'est-à-dire, à défaut de le faire bouger de sa place, à le traiter avec un outil symbolique, la parole.

Sans nier la dimension parfois vitale et urgente de la nécessité de la réponse, poser cet acte (de parole) s'oppose à la mise en œuvres d'actions quelles qu'elles soient et vise la jouissance, que le sujet ne perçoit pas, de l'impasse à laquelle il est parvenu ou de la plainte qu'il énonce inlassablement, jouissance qui, à perdurer dans la non reconnaissance, empêche tout repositionnement possible, c'est-à-dire toute désemprise du sujet des signifiants maître de cet Autre social qui veut que rien ne se modifie du côté de ce qui l'aliène ou de ce qui le représente, à savoir être un « exclu ».

Freud a d'emblée porté sa pratique hors de son cabinet : j'ajouterai que la complexité de ces rencontres cliniques « hors-cure » porte éclairage sur certaines difficultés cliniques auxquelles les cures nous confrontent. Je fais référence ici au fait que les processus au croisement du social et du sujet que nous tentons de définir infiltrent la subjectivité des personnes prises dans leurs méandres mais aussi, bien entendu, les sujets apparemment éloignés de ces problématiques : ce point appuie l'hypothèse évoquée plus haut sur la destruction des conditions même de la symbolisation, pour chacun. La précarité qui balaye tous les champs, économique, social, psychique, affectif, met les sujets en double errance, le glissement infernal que l'on connaît bien (perte d'emploi, ruptures familiales, perte de logement, rue... ) et l'objectalisation de la demande qui, dans une dimension de réduction caricaturale du désir fait coller les sujets au discours qui y gouverne dans une sorte de consensus absolu pour une substitution de l'objet de jouissance à l'aliénation symbolique.

Melman parle de " nouvelle économie psychique 8 », Lebrun de " mutation du lien social ${ }^{9}$ ».

Cette transformation radicale que nous évoque le télescopage de ce nouveau psychisme et de ce nouveau lien social, semble être à la fois effet et origine de cette destruction et a une portée clinique centrale : il ne s'agit plus de tenter de raccrocher les sujets qui dérivent à du symbolique qui serait sorti

8. C. Melman ; J.-P. Lebrun, L'homme sans gravité, Paris, Denoël, 2002.

9. J.-P. Lebrun, «Incidences de la mutation du lien social sur l'éducation », Le débat, nov.-déc. 2004. 
indemne de la mutation, mais bien d'offrir la possibilité de reconstruire, dans le transfert, une fiction, voire un trauma qui accueillerait l'élaboration du sujet quant au rapport double qu'il entretient avec la jouissance.

Dans les circuits sociaux, de ce sujet là il n'est pas question ; au mieux un objet réel viendra saturer une demande non identifiée, ce sont ces patients précairement logés, a minima soignés, illusoirement désintoxiqués qui, dans la confrontation au vide que nul objet ne saurait remplir, se suicident, accentuant le désarroi et le renoncement des équipes. Au pire, la rue restera le seul lieu où nous pourrons rencontrer des sujets enfermés dehors et tenter le pari du transfert qui soutiendrait un discours d'auteur.

Les psychanalystes convoqués dans ces institutions avec ou sans murs sont peut-être encore les seuls à n'être pas tout à fait captifs des exigences politiques en matière de satisfactions objectales de sujets réduits de la sorte à être des demandeurs, voire des revendicateurs, d'objets réels. N'ayant pas de réponse à produire dans la réalité, ils peuvent, dans le paradoxe de ces lieux où ce n'est plus la parole qui commande, ne pas céder sur leur engagement subversif de travail sur l'énonciation d'une langue aux manifestations désincarnées, sachant qu'il faut du temps pour y rencontrer l'énonciateur, le temps pour ce dernier d'y retrouver, dans le transfert, des bribes de ce qui constitue la « vérité » de son être.

Un lieu vidé de la parole est mortifère et devient rapidement inhabitable pour les sujets que l'on peut encore y rencontrer. C'est l'incarnation de ce réel que l'on tente de cerner, il contient bien, au bout du parcours, la mort. Il est aussi le champ de toutes les mises en place perverses qui trouvent là un espace de choix pour s'exercer puisque n'importe quelle figure pourra sembler préférable au vide.

Ici, il arrive que nous rencontrions les sujets, dans les mailles des filets tissés conjointement par la perversion des liens sociaux et les gouffres mélancoliques dans lesquels ils s'évanouissent.

Un projet de réhistoricisation peut s'appuyer sur l'idée qu'un ensemble vide n'en demeure pas moins un ensemble et que, n'étant pas dans le champ de la psychose, malgré la "psychotisation » qu'engendre les processus de démétaphorisation à l'œuvre, nous pouvons nous accrocher à son bord.

À la manière d'une tangente qui file sur ce bord, témoins furtifs d'une dépétrification non moins furtive des signifiants du sujet, notre acte de présence se justifie et pourra (re)devenir acte de parole, si l'on accepte de se laisser surprendre par nos propres signifiants " gelés 10 », c'est-à-dire notre propre « noyau psychotique ».

10. Pour reprendre une expression de Catherine Kolko. 
C'est le " hors-discours » des sujets rencontrés qui nous amène à évoquer la psychose et c'est ces tableaux qui ajoutent à la difficulté de l'instauration du transfert.

Cependant, à défaut d'occuper ces lieux, une clinique du vide pourrait continuer de voir se déliter des sujets même plus concernés par l'alternative de la vie ou de la mort. Le « hors-lieu » c'est le hors-sujet. La rencontre vise à mettre à distance le point d'horreur en restaurant le lien à l'autre, Autre différent de celui qui l'assigne à ce point critique.

CLINIQUE DES MÉTAMORPHOSES DU RÉEL

L'idée de métamorphose n'a pas la brutalité kafkaïenne mais œuvre insidieusement : $c^{\prime}$ est ce dont témoigne quinze ans de travail dans le champ dit «social » qui, nous allons voir comment, n'en demeure pas moins et doit demeurer le champ du sujet. Ce qui peut nous induire sur la pente de l'effacement de ce dernier, en écho aux processus qui lui échappent mais qui l'atteignent profondément c'est peut-être que, face au réel décrit, c'est-à-dire désinscrits d'un non-lien social, le sujet n'oppose plus forcément un symptôme, ce qui aurait maintenu le symbolique comme lieu à partir duquel notre éthique du désir d'analyser opère, mais un clivage où tantôt il réagit purement et simplement aux à-coups du réel sans que rien ne s'en inscrive pour lui, tantôt rien de ce réel ne peut se repérer et a fortiori s'énoncer, y compris comme tel.

Les analystes peuvent perdre leurs références lorsque les gens perdent les leurs... au sein de cette clinique de l'extrême.

Une jouissance non barrée de part et d'autre (sujet singulier et social) reflète (en même temps qu'elle s'y enracine) un réel, qui, de n'être pas nommé, ne révèle pas des troubles mais des désespoirs, et envahit l'espace de la coupure métaphorique dont sont garants les noms du père (et l'on sait bien que l'Autre social en est une figure capitale), invalidant du même coup la langue maternelle dans sa dimension de devoir énoncer ce qu'il en est du désir.

"La langue maternelle ce n'est pas la langue de la mère mais celle du désir de la mère 11 ", nous confiait Jacques Hassoun. Nous pourrions en déduire que son énonciation contient ce que l'enfant pourra intégrer de la castration, c'est-à-dire comment il s'arrangera à son tour de son rapport à la jouissance et à la loi.

11. Conversation avec Jacques Hassoun. 
Un sujet, c' est un signifiant, c'est-à-dire un effet de la parole. Nous avons à mettre en questionnement les signifiants du social qui dans leur remise en cause fondamentale des valeurs symboliques pétrifient le sujet en deçà de son émergence signifiante, en panne d'arrimage au lieu de l'Autre qui, de par les signifiants qu'il véhicule, "exclus », " précaires », " toxicos »... l'assigne dans la fixité du réel plus qu'il ne lui laisse accès à un " trésor ». Nous sommes tous inscrits dans une dépendance aux signifiants de l'Autre social, reste à savoir s'ils contiennent de quoi nous rendre présentables à un autre signifiant, c'est-à-dire de quoi nous rendre présentables au monde. Là, une clinique des sujets avec lesquels il nous faut faire le pari que les rencontrer dans une offre de parole les instituera comme sujets de demande ; c'est-à-dire ces personnes qui ne viennent rien demander, qui ne se plaignent ni ne revendiquent, témoignent de la confrontation à un univers chaotique où se succèdent ruptures et chutes, plus qu'à un monde où ils seraient " accueillis » dans leur singularité, c' est-à-dire un monde lui-même " présentable ».

En effet, deux cas de figure au moins se présentent. D'une part la déprivation winnicottienne où le sujet reste arrimé à ce qu'il donne à entendre comme un trauma réduisant la problématique psychique à une carence d'objet réel et faisant de ce " trauma » la cause de sa souffrance. Il va s'agir d'offrir l'espace de construction nécessaire à une remise en mouvement des représentations, des affects, des identifications, des espaces psychiques afin que ce réel qui ici se repère (même s'il ne s'énonce pas tout à fait pour ce qu'il est) puisse s'inscrire et faire trace. Cette hypothèse s'incarne cliniquement par la proposition qu'une trace s'inscrive justement, une reconstruction de la " scène » traumatique en quelque sorte. Que le fait qu'il lui arrive quelque chose interrompe une certaine jouissance du réel et se transforme en une certaine jouissance de la plainte, c'est-à-dire prenne les allures du symptôme auquel chaque sujet a droit : là se loge peut-être le véritable " droit commun ", pouvoir se débrouiller de son symptôme, c'est-à-dire en jouir ou en traduire la langue, mais au moins y prétendre.

D'autre part, cette sorte de "repli autistique » où plus rien, pas même sur un mode défensif, ne s'énonce. Ce sont ces sujets silencieux, figés, privés de leur demande et qui sont depuis longtemps dans des processus d'exclusion d'eux-mêmes, ces situations proches du néant auquel est associée la face mortelle de la pulsion qui nous conduisent à susciter, paradoxalement, une émergence du réel justement. Le silence qui perdure enferme le sujet en deçà de la parole, en deçà du symbolique. L'en-deçà du symbolique n'est pas, ici encore, sans évoquer le champ psychotique dans lequel les sujets sont confrontés à une jouissance qui fait retour dans le corps ou au vide mélancolique de la non-épreuve du désir. Faire émerger du réel semble avoir des effets traumatiques et nous replace dans le premier cas de figure, celui où le 
sujet devient, au lieu de rien, l'objet de consolation de sa propre perte. Proposer la construction, non pas d'un mythe mais d'une fiction à cet endroit consiste à extraire le sujet de l'impossibilité de se représenter ce qui est en train de lui arriver.

Autrement dit, en place de vide ou d'impossible au sens de ce qui revient toujours à la même place, si rien ne s'énonce, il nous faudrait en passer par la construction d'un trauma et si ça s'énonce comme réel mais ne s'inscrit pas, en passer par la construction d'un fantasme, entre jouissance et désir, qui, et c'est la fonction du fantasme, aura pour effet de relancer le désir.

Le travail clinique qui prend pour axe paradigmatique la psychanalyse, convoqué dans ces lieux d'errance ne s'enracine, pas plus que le sujet luimême, ni dans un dedans, ni dans un dehors. Seule la construction théoricoclinique de ce type d'hypothèses peut créer un espace de transfert, transfert dont il nous faut souvent prendre l'initiative dans la rencontre, créer un lieu habitable pour les signifiants enfouis de l'autre mais dont on prend le risque de penser l'existence.

« L'expérience de la pauvreté »n'est pas « une pauvreté de l'expérience " : ici s'ébauchent les prémisses d'une nouvelle approche du lien social.

Une fois cette dynamique symbolique, métaphorique remise en mouvement, encore faut-il être là pour qu'elle fasse événement-sujet, et sachant que son émergence ne se soutient pas toujours d'une temporalité, une fois que nous avons quelque chose à "voir ", nous pouvons envisager logiquement un temps «pour comprendre».

Là le sujet doit élaborer et assumer le fait qu'il n'est pas absent de sa rencontre avec la jouissance. C'est ce qui pourra nous mettre sur la voie de la reconnaissance d'une mise en jeu fantasmatique.

Comment le sujet s'approprie-t-il l'exclusion pour en faire le traitement de sa tragédie personnelle?

Comment le sujet peut-il se mettre en exclusion ? Les questions : " Comment en suis-je arrivé là ? » ou « comment ai-je pu supporter quelque chose qui me plongeait pourtant dans l'horreur? » relèvent d'une extraction du réel et d'une remise en circulation des chaînes signifiantes où le sujet pourra peut-être commencer à se dire entre les mots.

\section{BIBLIOGRAPHIE}

GORI, R. 2004. «L'enfant mort des passions », Cliniques méditerranéennes, 70, 45-58.

LACAN, J. 1966. Écrits, Paris, Le Seuil.

LACAN, J. 1966-1967. La logique du fantasme, séminaire inédit. 
LEBRUN, J.-P. 2004. «Incidences de la mutation du lien social sur l'éducation » Le débat. Melman, C. ; Lebrun, J.-P. 2002. L'homme sans gravité, Paris, Denoël.

\section{Résumé}

Quels sont les processus psychiques à l'œuvre dans le champ du réel social ? De quelles particularités témoigne aujourd'hui une clinique du réel social ? Que peuvent proposer les analystes sur ces terrains, d'une possible création de transfert dans laquelle s'élaboreraient des « traumas » et se symboliseraient des éléments d' « impossible»?

À partir d'une pratique clinique sur les terrains de la grande précarité, de l'exclusion et de la toxicomanie de rue, ce texte élabore une réflexion sur la nécessité du symptôme.

Mots-clés

Réel, lien social, désymbolisation, traumatisme, symptôme, exclusion.

\section{SOCIAL RACKINGS AND DESUBJECTIVATION}

\section{Summary}

Which are the psychic processes in the field of social reality? To which characteristics does testify today a private clinic to social reality?

What can propose the analysts on these grounds, of a possible creation of transfer in which « traumas » would be worked out and elements of « impossible » would be symbolized?

From a clinical practice on the grounds of the great precariousness, the exclusion and the drug-addiction of street, this text works out a reflexion on the need for the symptome.

\section{Keywords}

Reality («réel»), social bond, desymbolisation, traumatism, symptome, exclusion. 